literature with regard to the anesthetic requirement in patients with traumatic brain injury (TBI). The aim of our study was to determine the dose of propofol required for induction of anesthesia in patients with different grades of TBI undergoing surgery.

Materials and Methods: This prospective, observational study included patients with mild-, moderate-, and severe-grade TBI undergoing surgery within 48 hours of injury. Baseline bi-spectral index (BIS) values were recorded on injured and normal sides using bilateral BIS sensor. Anesthesia was induced with "a constant rate infusion of propofol using a target-controlled infusion (TCI) pump." Once the BIS value reached $\leq 40$, plasma site (Cp) and effect site (Ce) concentration and total dose of propofol required were noted from the TCI pump. Bilateral BIS and GCS at the end of surgery and GCS at discharge were also noted.

Results: Of the 96 patients, 27, 36, and 33 patients belonged to mild, moderate, and severe head injury, respectively. Baseline BIS on the injured side were 80,71 , and 55 , and on uninjured side were 89,81 , and 65 in mild, moderate and severe head injury. This difference between normal and injured side was statistically significant $(p<0.001)$ in each group. The Ce of propofol at induction in mild, moderate, and severe head injury were $6 \pm 0.9,5.82 \pm 0.98$, and $4.47 \pm 1.4 \mu \mathrm{g} / \mathrm{mL}(p<0.001)$, and dose of propofol required was $1.9 \pm 0.2,1.8 \pm 0.4$, and $1.41 \pm 0.5 \mathrm{mg} / \mathrm{kg}$, respectively $(p<0.001)$.

Conclusions: BIS values at baseline were significantly different between the groups on both sides. Dose of propofol required for induction of anesthesia was significantly lower in patients with severe head injury when compared with mild and moderate head injury.

\section{A0020 An Unusual Case of Acute Trigeminal Neuralgia Attack Precipitating Acute Heart Failure and Subsequent Anesthetic Management \\ Rajesha Srinivasaiah, ${ }^{1}$ Anshul Yadav, ${ }^{1}$ Shehla Shakooh, ${ }^{1}$ \\ Sandeep Lakhani ${ }^{1}$ \\ ${ }^{1}$ The Walton Centre NHS Foundation Trust, Liverpool, United Kingdom}

Background: Trigeminal neuralgia is a neuropathic pain disorder characterized by episodes of paroxysmal electric shock-like pain in the distribution of one or more divisions of trigeminal nerve. Cardiac symptoms like syncope associated with trigeminal neuralgia have been reported before. We report a case of patient whose acute trigeminal neuralgia attack precipitated an acute heart failure.

Case Description: An 82-year-old woman was posted for microvascular decompression (MVD) of trigeminal nerve for trigeminal neuralgia. Her past medical history included hypertension and mild mitral regurgitation. The preoperative investigations, including 2D echo, were essentially normal. Two days before the surgery, patient developed acute exacerbation of trigeminal neuralgia and within 2 hours developed breathlessness which was due to an acute left ventricular failure. Echo revealed LVEF of 34\% with severe systolic dysfunction and severe MR. She was appropriately treated in CCU. Later she was reviewed by a neurosurgeon and anesthetist.
The decision was taken to change her treatment from MVD to percutaneous balloon compression (PBC) of TGN.

$\mathrm{PBC}$ involves cannulation of the foramen ovale, followed by inflation of a Fogarty balloon in Meckel's cave under fluoroscopic guidance to damage retrogasserian trigeminal fibers. It is a short-duration, minimally invasive procedure done as a day care case under GA. Trigeminal neuralgia attacks are amongst the most painful conditions known. Acute pain can cause activation of sympathetic nervous system and increased catecholamine levels resulting in increased cardiac work. It also causes activation of the renin-angiotensin-aldosterone system leading to fluid retention and overload.

Conclusions: Cardiac symptoms are very rare manifestations of trigeminal neuralgia. Balloon compression of trigeminal nerve is an important and useful treatment option for patients with trigeminal neuralgia in elderly and frail people when other forms of more invasive treatments are not suitable.

\section{A0021 To Study the Outcome of Massive Transfusion in Pediatric Patients Undergoing Elective Craniotomy: \\ A Retrospective Descriptive Study \\ Swati Patel, ${ }^{1}$ Ramamani Mariappan ${ }^{1}$ \\ 'Department of Anesthesia, Christian Medical College, Vellore, Tamil Nadu, India}

Background: Children undergoing surgical resection of vascular tumor and malformations are at a great risk of rapid, massive blood loss. The primary goals while managing these children who need massive transfusion are avoiding hypotension, maintaining adequate CPP and oxygenation. Goals are achieved by transfusing appropriate volume of blood and blood products at an appropriate time to facilitate faster recovery with minimal complications.

Materials and Methods: Children who received blood transfusion during the elective craniotomy over a period of 5 years were taken from the computerized hospital in-patient data source (CHIPS), of whom, those who had > 50\% volume loss in 3 hours were analyzed. Out of 140 children who received transfusion during elective craniotomy, a total of 26 children received massive transfusion. All charts were analyzed in detail. Patient demographics, preoperative, intraoperative factors were correlated with postoperative factors to predict the morbidity and mortality.

Results: Demographics (median and interquartile range [IQR]): Age 4 (2-12) years; weight $14(11-30) \mathrm{kg}$; height 91 (46-136) cm; BMI 13 (9-17) kg/m², male-to-female ratio $16: 10$. Four out of 26 children had congenital anomalies. Intraoperative parameters: Average preoperative Hb was (median IOR) 10 (8-12) g/dL. 16/26(61\%) received inhalational anesthetic and 10/26 (39\%) received both inhalational and TIVA for maintenance. Regarding the IV access, 25/26 (96\%) of children had two peripheral lines along with one central line. 12/26 (46\%) children received tranexamic acid. Average blood loss was $52.5 \pm 34.5 \mathrm{~mL} / \mathrm{kg}$. 14/26 (53\%) children had intraoperative hemodynamic instability who needed noradrenaline, 9 had no hemodynamic instability, and 1 had 
severe instability and needed both adrenaline and noradrenaline. Fourteen (54\%) of 26 children had metabolic acidosis, and more than $30 \%$ patients had electrolyte abnormality of which hypocalcemia was the commonest followed by hypokalemia. Average amount of PRBC transfusion was 40.7 $\pm 26.5 \mathrm{~mL} / \mathrm{kg}$; WBC $25.7 \pm 12.7 \mathrm{~mL} / \mathrm{kg} ;$ FFP $20.5 \pm 12.8 \mathrm{~mL} /$ $\mathrm{kg}$; platelets $7.7 \pm 4.05 \mathrm{~mL} / \mathrm{kg}$; and cryo $4.77 \pm 2.32 \mathrm{~mL} / \mathrm{kg}$. 3 patients $(22.5 \%)$ had mild transfusion reaction and needed antihistamine and steroids. Postoperative parameters: Out of 26 patients, 11 (42.3\%) were electively ventilated for average of 23 hours. $6 / 26$ patients (23.1\%) were on inotropic support for 10 to 21 hours. Average days of ICU and hospital stay were 1.5 and 10 days, respectively. 11/26 had postoperative complication. There was no death reported in this series.

Conclusions: Anticipation, preparation, and aggressive intraoperative resuscitation with blood and blood products during massive blood loss reduce the postoperative morbidity and mortality.

\section{A0022 Effect of Remote Ischemic Preconditioning on Cerebral Vasospasm and Biomarkers of Cerebral Ischemia in Aneurysmal Subarachnoid Hemorrhage}

Sangeetha R. Palaniswamy, ${ }^{1}$ Venkatapura J. Ramesh, ${ }^{1}$ Sriganesh, ${ }^{1}$ Rita Christopher, ${ }^{1}$ Dhananjaya Bhat ${ }^{2}$

${ }^{1}$ Department of Anaesthesia, National Institute of Mental

Health and Neurosciences, Bengaluru, India

${ }^{2}$ Department of Neurosurgery, National Institute of Mental Health and Neurosciences, Bengaluru, India

Background: Cerebral vasospasm is a dreaded complication of aneurysmal subarachnoid hemorrhage (aSAH) predisposing one to delayed cerebral ischemia. We investigated the cerebroprotective effects of remote ischemic preconditioning (RIPC) in patients with aSAH.

Materials and Methods: This was a single-center, prospective, parallel-group, randomized, pilot trial, approved by institutional ethics committee. Patients with aSAH admitted to National Institute of Mental Health and Neurosciences for surgical clipping, fulfilling the trial inclusion criteria, were randomized to true RIPC ( $n=12$ ) (inflating upper extremity blood pressure cuff thrice for 5 minutes to $30 \mathrm{~mm} \mathrm{Hg}$ above systolic blood pressure) or sham RIPC $(n=12)$ (inflating blood pressure cuff thrice for 5 minutes to $30 \mathrm{~mm} \mathrm{Hg}$ ) in a 1:1 allocation ratio using a computerized allocation sequence and block randomization. Our outcome measures, assessed by an observer blinded to the study intervention, were vasospasm on cerebral angiography and transcranial Doppler (TCD) study, and serum concentration of biomarkers of astrocytic and neuronal damage S100B and NSE at 24 hours after RIPC and on day 7 of ictus.

Results: Incidence of vasospasm on angiography ( $n=13$ ) was $25 \%$ (1/4 patients) in RIPC group and 89\% (8/9 patients) in sham group ( $p=0.052$ ). Vasospasm on TCD study was diagnosed in $1 / 13$ patients (7.7\%) and $4 / 12$ patients (33.3\%) in true and sham RIPC groups, respectively ( $p=0.16$ ). There was no difference in serum concentrations of S100B and NSE between groups ( $p=0.56$ and 0.31 , respectively ) and over time in either groups ( $p=0.318$ and 0.494 for S100B and $p=0.661$ and 0.174 for NSE in RIPC and sham groups, respectively). None had adverse effects on transient limb ischemia with RIPC.

Conclusions: This pilot trial showed that RIPC is feasible and safe. Our preliminary findings suggest a protective role of RIPC in prevention of vasospasm after aSAH, which needs confirmation by a larger trial.

A0023 Effect of Perioperative Hemoglobin Concentration on Cerebral Oxygen Extraction and Neurological Outcome following Aneurysmal Subarachnoid Hemorrhage

Deepak Rajappa, ${ }^{1}$ Hemant Bhagat, ${ }^{1}$ Nidhi Panda,

Ankur Luthra, ${ }^{1}$ Ashish Aggarwal ${ }^{2}$

${ }^{1}$ Department of Anaesthesia and Intensive Care, Postgraduate Institute of Medical Education and Research, Chandigarh, India ${ }^{2}$ Department of Neurosurgery, Postgraduate Institute of Medical Education and Research, Chandigarh, India

Background: The decrease in hemoglobin concentration ( $\mathrm{Hb}$ ) levels has a better rheological and cerebral perfusion spectrum. However, reduced Hb levels are associated with decreased cerebral oxygenation as a result of reduction in oxygen carrying capacity. On the contrary, higher Hb levels compromise blood rheology and cerebral perfusion. Optimal $\mathrm{Hb}$ in subarachnoid hemorrhage (SAH) patients is still controversial for better outcome of the SAH patients. The study aimed to evaluate the effect of perioperative $\mathrm{Hb}$ levels on cerebral oxygen extraction and neurological outcome in SAH patients.

Materials and Methods: Patients undergoing aneurysmal clipping for ruptured SAH were included. After anesthesia induction and following placement of jugular venous catheter, patients were additionally monitored for partial pressure of oxygen in arterial blood $\left(\mathrm{PaO}_{2}\right)$, saturation in arterial blood $\left(\mathrm{SaO}_{2}\right)$, saturation in jugular venous blood $\left.(\mathrm{SjvO})_{2}\right)$, and $\mathrm{Hb}$. Samples were obtained hourly in the intraoperative period and twice daily for 3 days in postoperative period to know $\mathrm{Hb}$ and cerebral oxygen extraction. Neurological outcome of the patients was measured with mRS and GOS-E at 7 days and 3 months, respectively. Hb values were correlated with arteriovenous difference in oxygenation $\left(\mathrm{AVDO}_{2}\right)$ and neurological outcome.

Results: Eighty patients were included in the study. Significant correlation was obtained between intraoperative and postoperative $\mathrm{Hb}$ values with GOS-E at 3 months $(p<0.05)$. No correlation was seen between $\mathrm{Hb}$ and MRS at 7 days. There was a positive linear relationship between $\mathrm{Hb}$ and $\mathrm{AVDO}_{2}$ values $(p<0.001)$. Multivariate analysis showed intraoperative $\mathrm{Hb}<10 \mathrm{~g} / \mathrm{dL}$ as an independent risk factor for poor neurological outcome.

Conclusions: Perioperative $\mathrm{Hb}$ values correlate with GOS-E at 3 months. Mean $\mathrm{Hb}$ of $<10 \mathrm{~g} / \mathrm{dL}$ during perioperative period appears to have an unfavorable neurological outcome at 3 months following aneurysmal SAH. 